Voix et Images

volxetimages

\title{
Nelligan livré aux fauves de l'interprétation
}

\section{Roger Chamberland}

Volume 18, numéro 3 (54), printemps 1993

Littérature, folie, altérité

URI : https://id.erudit.org/iderudit/201052ar

DOI : https://doi.org/10.7202/201052ar

Aller au sommaire du numéro

Éditeur(s)

Université du Québec à Montréal

ISSN

0318-9201 (imprimé)

1705-933X (numérique)

Découvrir la revue

Citer cet article

Chamberland, R. (1993). Nelligan livré aux fauves de l'interprétation. Voix et Images, 18(3), 590-593. https://doi.org/10.7202/201052ar d'utilisation que vous pouvez consulter en ligne.

https://apropos.erudit.org/fr/usagers/politique-dutilisation/ 


\section{Nelligan livré aux fauves de l'interprétation}

\section{Roger Chamberland, Université Laval}

Ces dernières années ont été particulièrement fertiles en collections d'articles publiées par des universitaires qui ont principalement fait leur marque durant les années soixante et soixante-dix. Selon toute apparence, l'heure des bilans est arrivée au seuil du retrait de la vie universitaire; il faut penser à la relève, à ce qui pourrait servir de legs à la critique québécoise: des articles difficiles d'accès ou introuvables, des conférences non publiées, des textes écrits dans le feu de l'action mais demeurés inédits et des chapitres de livres qui, amalgamés aux restes des essais, permettent de composer un portrait de l'intellectuel qui, dans bien des cas, a connu et endossé des modes analytiques partant du structuralisme à la sémiotique allant par la thématique, la socio-critique, la psychocritique et ainsi de suite. Après les essais critiques de Jean Marcel, Laurent Mailhot, André Brochu, Joseph Bonenfant, Robert Giroux, Michel van Schendel, pour n'en nommer que quelques-uns, voilà que Réjean Robidoux nous offre les siens qui ont, à la différence de plusieurs autres, pour caractéristique de porter sur un seul sujet - et il est de taille - Émile Nelligan.

Contrairement à plusieurs de ses collègues universitaires, Robidoux a toujours maintenu une seule ligne de pensée critique, du moins si l'on en juge par les articles et les textes rassemblés ici sous le titre de Connaissance de Nelligan ${ }^{1}$. Sa fidélité à la critique lansonienne - et il ne s'en cache pas dans sa préface - l'expose plus que quiconque à la polémique.

Je sais par ailleurs que mon écriture est résolument universitaire et qu'elle met en branle, à l'ancienne mode, tout le lourd appareil tant décrié (notes, références, documentation historique, analytique, factuelle, terre à terre...), que j’ai le mauvais goût de juger nécessaire pour réduire au minimum cette part monstrueuse de hasard que je reproche opiniâtrement aux prétendus experts de déployer pour connaître Nelligan (p. 10).

Doutera-t-on par ailleurs de sa sincérité et de son engagement envers Nelligan, lui qui, depuis les débuts de sa carrière, s'y est intéressé et a participé à l'édition critique des Poésies complètes avec Paul Wyczynski?

L'ouvrage est divisé en quatre parties, les deux premières étant consacrées à l'œuvre de Nelligan, à son message, à son mode d'écriture, à ses caractéristisques fondamentales; les deux dernières étant le 
fruit du travail du releveur de torts, de celui dont le savoir ponctuel, circonstancié, anecdotique sera toujours plus fort que la pure spéculation et l'hypothèse conjoncturelle. Comme l'indique le titre de la première section, "L'opus mirandum de Nelligan", c'est l'œuvre à admirer. Dès le premier texte, "Expérience et création", Robidoux donne à fond dans l'apologie et explique l'œuvre par la vie: "Toute l'expérience poétique de Nelligan est caractérisée par une névrose d'angoisse qui. le pousse irrésistiblement à la perte de soi" (p. 27). Mais cette expérience poétique n'a pas été totale puisque "le poète s'est perdu avant de toucher parfaitement le sommet obligé de toute entreprise de connaissance. (p. 43), telle que définie par Rimbaud et Novalis, et qui implique que "la reproduction de l'abșolue harmonie du monde doit aussi trouver son expression dans la mise en place d'un univers qui transcende le moi "(ibid.). Même si Nelligan ne répond pas entièrement aux exigences qui lui permettraient d'être considéré comme un poète accompli, du moins peut-il faire partie des "grands" parce qu'il est le premier à s'être rendu si loin dans les exigences de la création. Le deuxième texte, "La signification de Nelligan", reprend cette même idée, mais suggère que la véritable signification de Nelligan serait celle que nous livrerait un "psychiatre qui se doublerait d'un critique littéraire ". Ce dernier pourrait suivre l'évolution du mal en regard de celle des thèmes; on aurait ainsi l'ordre véritable d'écriture des poèmes. À défaût du spécialiste rêvé, Robidoux suggère plutôt que Nelligan a su capitaliser sur son déséquilibre pour vivre son rêve et l'écrire jusqu'à le prendre pour la réalité et s'y abolir. Dès lors, "l'œuvre et la vie sont soudées dans une aventure singulière et parfaitement identifiée. (p. 54). Dix ans plus tard, dans le dernier texte de cette section, le critique récidive dans sa volonté de faire coïncider à tout prix l'œuvre et la vie nelliganiennes en y ajoutant cette fois-ci une touche sociocritique afin de montrer que Nelligan "a assumé un rôle exemplaire face à notre collectivité" (p. 75). De telles propositions, pour intéressantes qu'elles soient en surface, sont néanmoins décevantes parce qu'elles réduisent l'accès à l'œuvre et l'érigent selon une organisation systémique du delirium tremens. Mais cela nous conduit où au juste? À considérer que chaque poème de Nelligan est une énigme compréhensible uniquement en fonction de tel ou tel autre fait, événement, circonstance ou personne qui seraient intervenus au moment précis de l'écriture du texte ${ }^{2}$.

Dans la deuxième partie, l'auteur s'intéresse plus encore au “texte nelliganien.. Il en étudie la prosodie puis rend compte du travail, de la discipline et des exigences qu'il s'est imposés lors de la confection de l'édition critique. Dans "Mythe et réalité", la troisième division, 
l'auteur prend à contre-pied les "impies " et les pourfendeurs, ceux qui délaissent la critique historique au profit d'une interprétation plus libre. Des victimes, il y en a beaucoup: Jean Larose, Bernard Courteau, contre qui deux textes sont dirigés, Michel Tremblay et André Gagnon. En somme, il existe une orthodoxie interprétative quant à la lecture de Nelligan qui lie, de façon excessivement étroite, les données biographiques et l'œuvre, même si des épisodes de la vie sont encore obscurs, même si le diagnostic médical quant à la "folie " de Nelligan est, aux dires de Robidoux, inattaquable parce qu'il a été fait par des médecins consciencieux dont on ne peut mettre en doute l'expertise. Pourtant quelle était la connaissance médicale eu égard aux maladies mentales? Doit-on rappeler que l'on avait l'enfermement facile il n'y a pas encore si longtemps? Que les témoignages faits par ceux que l'on nomme "les enfants de Duplessis" sont accablants et profondément troublants? Nelligan n'aurait-il pas pu être une victime malheureuse d'une société pour qui le moindre écart comportemental était le signe d'une déviance psychologique? On peut maintenir un doute raisonnable sur l'institution psychiatrique québécoise du début du siècle et, surtout, relire l'Histoire de la folie à l'âge classique de Michel Foucault dont les analyses peuvent alimenter notre scepticisme.

Le premier texte de la quatrième partie, qui devait être consacrée à "Nelligan et Dantin", prolonge toutefois la mise au ban d'auteurs qui ont voulu faire de la fiction autour du poète maudit. Le grand tort du duo Aude Nantais et Jean-Joseph Tremblay, les auteurs de Portrait déchiré de Nelligan, une fiction dramatique, est d'avoir lu et interprété un document du dossier médical de Nelligan conservé à Saint-Jean-deDieu, dans lequel on stipule que le patient a subi l'ablation des "principales". À partir de ce fait, Nantais-Tremblay convertissent Nelligan en homosexuel que l'on a dû opérer à cause de déviance sexuelle, thèse par ailleurs reprise et suggérée dans l'opéra de TremblayGagnon et dans le film de Robert Favreau. Mais voilà: là où le bât blesse, c'est quand les auteurs donnent libre cours à leur imagination, interprètent à leur guise ce qu'a pu être la vie de Nelligan au grand dam d'un Robidoux, consterné que l'on ne respecte pas plus, même dans la fiction, les données biographiques, certifiées authentiques!

Dans son texte de clôture, "Apologie de Dantin", le critique se porte, cette fois-ci, à la défense de Louis Dantin, celui-là même qui a été un lecteur privilégié et le premier éditeur de l'œuvre (connue) de Nelligan. Là-dessus, les spéculations vont bon train à savoir que Dantin aurait peut-être fait disparaître bon nombre d'inédits. L'auteur de Connaissance de Nelligan est catégorique: "L'idée seule que Dantin 
ait pu sciemment détruire des textes de Nelligan est une mesquinerie de l'esprit, en soi infamante. (p. 21), et il s'emploie à défendre Dantin contre ces "mythographes" et autres consorts.

On ne peut pas dire que nous ayons une meilleure connaissance de Nelligan après la lecture de ces onze articles, ni même que nous soyons plus en mesure de le comprendre et de l'interpréter. De fait, il devient même difficile de lancer une quelconque polémique, la position de l'auteur étant solidement ancrée non pas tant sur l'interprétation des textes que sur leur pleine et entière adéquation à la vie même de Nelligan. Dès lors, il devient difficile, par manque de ressources documentaires, de s'engager sur un terrain miné.

1. Rejean Robidoux, Connaissance de Nelligan, Montréal, Fides, 1992, 186 p.

2. C'est à cette lecture exégétique que s'est amusé Pierre H. Lemieux dans son essai Nelligan amoureux paru cette même année. 